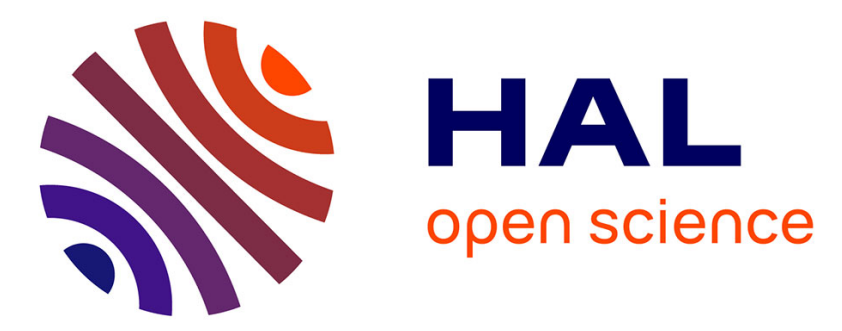

\title{
Highly porous scaffolds for Ru-based microsupercapacitor electrodes using hydrogen bubble templated electrodeposition
}

Lotfi Benali Karroubi, Sai Gourang Patnaik, Birhanu Desalegn Assresahegn, Botayna Bounor, Chau Cam Hoang Tran, Sakeb Hasan Choudhury, David Bourrier, Daniel Guay, David Pech

\section{To cite this version:}

Lotfi Benali Karroubi, Sai Gourang Patnaik, Birhanu Desalegn Assresahegn, Botayna Bounor, Chau Cam Hoang Tran, et al.. Highly porous scaffolds for Ru-based microsupercapacitor electrodes using hydrogen bubble templated electrodeposition. Energy Storage Materials, 2022, 47, pp.134-140. 10.1016/j.ensm.2022.02.009 . hal-03585171

\author{
HAL Id: hal-03585171 \\ https://hal.laas.fr/hal-03585171
}

Submitted on 23 Feb 2022

HAL is a multi-disciplinary open access archive for the deposit and dissemination of scientific research documents, whether they are published or not. The documents may come from teaching and research institutions in France or abroad, or from public or private research centers.
L'archive ouverte pluridisciplinaire HAL, est destinée au dépôt et à la diffusion de documents scientifiques de niveau recherche, publiés ou non, émanant des établissements d'enseignement et de recherche français ou étrangers, des laboratoires publics ou privés. 


\section{Highly porous scaffolds for Ru-based microsupercapacitor}

\section{electrodes using hydrogen bubble templated electrodeposition}

Lotfi Benali Karroubia, Sai Gourang Patnaika, Birhanu Desalegn Assresahegn ${ }^{b}$, Botayna Bounor ${ }^{a}$, Chau Cam Hoang Tran ${ }^{a}$, Sakeb Hasan Choudhurya, David Bourriera, Daniel Guay ${ }^{\mathrm{b},{ }^{*}}$, David Pech ${ }^{\mathrm{a},{ }^{*}}$

aLAAS-CNRS, Université de Toulouse, CNRS, 7 avenue du colonel Roche, Toulouse 31400, France

bINRS-Énergie, Matériaux et Télécommunications, 1650 boulevard Lionel Boulet, Varennes, C.P. 1020, Québec J3X 1S2, Canada.

"Corresponding authors : Daniel Guay (guay@emt.inrs.ca), David Pech (dpech@laas.fr)

\section{Abstract}

Microsupercapacitor electrodes with 3D architectures have drawn increasing interest in recent years due to their better energetic performances while maintaining a reduced footprint occupancy. Here, we report two different strategies to realize highly porous scaffolds of $\mathrm{RuO}_{2}$ on $\mathrm{Si}$ wafers, with areal enlargement factors exceeding 13000 $\mathrm{cm}^{2} / \mathrm{cm}^{2}$. The 3D structures are synthesized via hydrogen bubble dynamic template at low temperature, onto which a conformal electrodeposition of hydrated $\mathrm{RuO}_{2}$ thin film is performed. The microsupercapacitor electrode exhibits a record areal capacitance of $24 \mathrm{~F} \mathrm{~cm}^{-2}$ with an incredible areal energy of $8.7 \mathrm{~J} \mathrm{~cm}^{-2}($ i.e. $2.4 \mathrm{mWh} \mathrm{cm}-2)$, together with a high power of $354 \mathrm{~mW} \mathrm{~cm}^{-2}$ and an excellent cycling stability. These electrodes stand miles ahead from previously reported studies both in terms of their surface area and surface capacitance, making them suitable for loT applications demanding high energy/high power density per footprint area. 


\section{Introduction}

On board micro-energy storage is of significant interest for integration of independent autonomous microelectronic devices concerning the rapidly growing loT (Internet of Things)-based industry. Many of these devices have their own power/energy supply and management requirements depending on associated complexities in the electronics, and hence necessitate customized micro-energy supply as well. Microsupercapacitors feature high power density, extraordinary cycling stability and can either complement microbatteries or even be utilized as standalone power delivery devices [1]. However, the limiting characteristic of microsupercapacitors is their energy density. Constructing 3D architectures to maximize the surface area has been a long-standing strategy to increase the specific energy density while keeping in mind the limited footprint surface area available [2]. The technical challenge here is to maintain electronic conductivity, allow sufficient room for electrolyte percolation and have tunable parameters to manipulate the $3 \mathrm{D}$ structuring. In terms of feasibility of incorporation into a commercial microfabrication line, the process needs to be facile and readily scalable. With regards to materials engineering, compared to double layer charge storage, pseudocapacitive materials offer an alternative with extremely high energy density. The charge storage mechanism in these materials relies on closely overlapping surface redox reactions that do not involve any crystallographic phase change $[3,4]$.

Three-dimensional porous current collector constructed through dynamic hydrogen bubble template (DHBT) is an industrially compatible process that results in extremely large area enhancement factors (AEF) with tunable porosity and electrode thickness [5]. More importantly, this strategy can also be utilized to efficiently construct 3D metallic current collectors on silicon wafers, polymeric substrates and other soft 
templates by first coating a metallic thin film on them. We have previously showcased their enormous potential in the construction of varied electrode architectures for high power/energy microsupercapacitors $[6-8]$ and microbatteries $[7,9,10]$ with superlative performances. The choice of metal, level of porosity as well as thickness of the porous film in the DHBT process are important considerations as they govern the subsequent manipulations that these films can sustain without compromising their mechanical stability and surface area. Porous films of metals like Au quickly lose a fraction of their surface area upon an increase in temperature. This relatively lower stability of porous Au can be explained based on the very high surface diffusion coefficient of Au atoms and high driving force to reduce its surface energy leading to coarsening of the dendritic structure, especially at high temperatures [11,12]. This is an important drawback as thin film deposition of many pseudocapacitive active materials requires high temperature processing, and hence any electrode based on a 3D architecture to be used in conjugation with these materials should remain stable during the film deposition process. We have previously reported on the electrodeposition of thin films of hydrous $\mathrm{RuO}_{2}$ on conductive porous Au matrices with large surface area. Continuing along these lines, we report here on porous $\mathrm{Pt} / \mathrm{Cu}$ alloy current collectors with good thermal stability and a tremendous area enhancement factor (AEF) exceeding 13000 $\mathrm{cm}^{2} / \mathrm{cm}^{2}$. We then conformally deposit thin films of pseudocapacitive hydrous $\mathrm{RuO}_{2}$ materials, thus obtaining the highest capacitance ever reported for such systems, above $24 \mathrm{~F} \mathrm{~cm}^{-2}$. Following a similar strategy, we also demonstrate the fabrication of free-standing porous hydrous $\mathrm{RuO}_{2}$ based pseudocapacitive films without any scaffolds, which exhibit $\sim 2.5 \mathrm{~F} \mathrm{~cm}^{-2}$ for a lower AEF of $690 \mathrm{~cm}^{2} / \mathrm{cm}^{2}$. Our results show that, with synthetic flexibility to tune the porosity and thickness, DHBT can definitely serve as an ideal platform for the fabrication of microsupercapacitors with extremely 
high energy/power density. Most importantly, the entire fabrication process is based on the use of aqueous electrolytes and does not involve vacuum-based deposition techniques. This is a cost-effective and industrially feasible approach towards the fabrication of high-energy, high-power microdevices with exceptional stability and design flexibility.

\section{Materials and methods}

\subsection{Fabrication of the $3 D$ porous $P t$}

$\mathrm{A} \mathrm{Ti}(50 \mathrm{~nm}) / \mathrm{Pt}(200 \mathrm{~nm})$ thin film was deposited by evaporation on an oxidized silicon substrate and electrochemically pretreated by cycling the potential at a scan rate of $100 \mathrm{mV} \mathrm{s}^{-1}$ between -0.3 and $+1.7 \mathrm{~V}$ versus a saturated calomel reference electrode (SCE) in $0.1 \mathrm{M} \mathrm{H}_{2} \mathrm{SO}_{4}$ until a stable voltammogram was obtained. The substrate was then cycled between -0.6 and +1.2 vs. SCE in an electrolytic solution of $\mathrm{H}_{2} \mathrm{PtCl}_{6}$ and $\mathrm{CuSO}_{4} .5 \mathrm{H}_{2} \mathrm{O}$ using different ratios in $1 \mathrm{M} \mathrm{H}_{2} \mathrm{SO}_{4}$ bath. This was followed by the application of a constant current of $-6.5 \mathrm{~A} \mathrm{~cm}^{-2}$ for different durations. Cu was further electrochemically de-alloyed through cyclic voltammetry in $0.5 \mathrm{M} \mathrm{H}_{2} \mathrm{SO}_{4}$ between -0.38 to +1.2 vs. SCE for 50 cycles at $10 \mathrm{mV} \mathrm{s}^{-1}$. The obtained sample was termed as $\mathrm{Pt}$ DHBT (Figure S1a).

\subsection{Conformal coating of hydrous $\mathrm{RuO}_{2}$ on Pt DHBT}

Electrodeposition of $\mathrm{RuO}_{2} \cdot \mathrm{xH}_{2} \mathrm{O}$ on $\mathrm{Pt}$ DHBT was carried out in a pseudo-buffer solution of $10 \mathrm{mM}$ solution of $\mathrm{RuCl}_{3} \cdot \mathrm{nH}_{2} \mathrm{O}$ in $0.2 \mathrm{M} \mathrm{HCl} / 0.2 \mathrm{M} \mathrm{KCl}$ at $50^{\circ} \mathrm{C}$ under constant stirring through cyclic voltammetry between -0.3 to $0.95 \mathrm{~V}$ vs. SCE at $10 \mathrm{mV}$ $\mathrm{s}^{-1}$ 


\subsection{Fabrication of self-standing $3 \mathrm{D}$ porous hydrous $\mathrm{RuO}_{2}$}

DHBT of Ru/Cu alloys were prepared using a constant current of $-7.5 \mathrm{~A} \mathrm{~cm}^{-2}$ for $5 \mathrm{~min}$ in an optimized bath consisting of $30 \mathrm{mM} \mathrm{RuCl}_{3} . \mathrm{nH}_{2} \mathrm{O}$ and $15 \mathrm{mM} \mathrm{CuSO}_{4} .5 \mathrm{H}_{2} \mathrm{O}$ in $1 \mathrm{M}$ $\mathrm{H}_{2} \mathrm{SO}_{4}$ with $1 \mathrm{~mL}$ of PEG (polyethylene glycol) to prevent bubble coalescence during the DHBT process. This yielded porous Ru/Cu films. Subsequently, Cu was de-alloyed from the porous film by performing cyclic voltammetry between 0 and $1.1 \mathrm{~V} v \mathrm{vs} . \mathrm{Ag} / \mathrm{AgCl}$ at $10 \mathrm{mV} \mathrm{s}^{-1}$ in $0.5 \mathrm{M} \mathrm{H}_{2} \mathrm{SO}_{4}$ for 10 cycles. This was followed by oxidation of Ru through cyclic voltammetry between -0.2 to $1.2 \mathrm{~V}$ vs. $\mathrm{Ag} / \mathrm{AgCl}$ in $0.5 \mathrm{M} \mathrm{H}_{2} \mathrm{SO}_{4}$ (Figure S1b).

\subsection{Material characterizations}

The morphological and elemental analysis of the electrodes surface were performed using a FEI Helios 600i field emission scanning electron microscope (SEM) equipped with an energy dispersive X-ray (EDX) detector. The crystallographic structures were analyzed by grazing incidence X-ray diffraction (GI-XRD) measurements on a Bruker D8 Advanced X-ray diffractometer with Cu Ka radiation (1.54184 Å), operating at 40 $\mathrm{kV}$ and $40 \mathrm{~mA}$. The surface chemical composition of electrodes was estimated via Xray photoelectron spectroscopy (XPS) using a VG Escalab 220i-XL instrument operating with a monochromatic Al Ka X-ray source (1486.6 eV). The electrochemical synthesis and characterizations were performed with a VMP-3 and a VSP Biologic potentiostat connected to an external $10 \mathrm{~A}$ booster channel.

\subsection{AEF factor calculation of Pt and Ru scaffolds}

To compare the increase in surface area to that of apparent geometrical surface area, the following metric named Area Enlargement Factor (AEF) is introduced:

$\mathrm{AEF}=$ Electrochemical Active Surface Area (EASA) / Geometrical Area 
The electrochemical surface area of porous Pt electrodes was calculated by integrating the charge of the hydrogen adsorption/desorption region observed by cyclic voltammetry in $0.5 \mathrm{M} \mathrm{H}_{2} \mathrm{SO}_{4}$ using a theoretical value of $\mathrm{H}_{\text {ads }}$ of $210 \mu \mathrm{C} \mathrm{cm}^{-2}$ [13]. Due to the overlap of the hydrogen and oxygen adsorption/desorption regions on $\mathrm{Ru}$ surfaces, the determination of the surface area of the Ru scaffold was undertaken by under potential deposition (UPD) of $\mathrm{Cu}$ in a solution of $10 \mathrm{mM} \mathrm{CuSO}_{4} .5 \mathrm{H}_{2} \mathrm{O}$ in $0.5 \mathrm{M}$ $\mathrm{H}_{2} \mathrm{SO}_{4}$ and using a Cu stripping charge of $420 \mu \mathrm{C} \mathrm{cm}^{-2}$ [14].

\section{Results and discussion}

\subsection{Porous Pt (Pt DHBT)}

It is known that the surface diffusion coefficient of $\mathrm{Pt}$ is $3-4$ orders of magnitude lower than $\mathrm{Au}$ [15]. Accordingly, the addition of Pt in small amount was used in the past as a strategy to stabilize porous Au/Ag films, as Pt atoms would accumulate at the surface of the films, mostly at the kink and step edge sites, thus helping to maintain the structural integrity of the films. Thus, it was inferred that porous Pt films would be less prone to coarsening and undergoing a reduction of their surface area, unlike in the case of porous Au films (Figure S2). First, we try to prepare porous Pt DHBT films by applying a constant negative current in concentrated acidic electrolytes. However, all initial attempts to fabricate pure Pt DHBT films were unsuccessful, films thus prepared showing poor mechanical stability and adhesion to the substrates. Hence, we use a different strategy by first preparing Pt-M alloy DHBT thin films that have good mechanical stability and showed excellent adhesion to the substrate, before performing an electrochemical de-alloying step of the less noble metal $\mathrm{M}$ to get a $\mathrm{Pt}$ porous films [16-18]. The choice of the second and less noble metal to be de-alloyed is critical, as it directly affects the creation of vacancies in the Pt films. Cu can be easily 
de-alloyed in mildly acidic medium and hence was chosen as the second metal to be alloyed with Pt. Initially, DHBT deposition at a constant negative current was performed using electrolytes with different $\mathrm{Cu}$ and $\mathrm{Pt}$ compositions, followed by de-alloying of $\mathrm{Cu}$ by cyclic voltammetry (CV) (Fig. 1a). With increasing de-alloying cycles, the Pt surface area starts to increase rapidly, as indicated by the increase in the $\mathrm{H}^{+}$ adsorption/desorption peaks with a decrease of $\mathrm{Cu}$ related peaks, before reaching steady-state after around 50 cycles. The combined use of a dynamic template and $\mathrm{Cu}$ de-alloying results in formation of a highly porous DHBT structure with extra-porosity at the nanoscale. Depositions for different durations were evaluated to examine the adherence of the films onto the substrate (Table S1 and Figure S3). The optimum ratio of $\mathrm{Pt}: \mathrm{Cu}$ (67:33\%, bath composition) yielded porous films with good stability, adherence and mechanical strength for up to $600 \mathrm{~s}$ of deposition. The AEFs of the Pt DHBT films were first evaluated by integrating the charge under the $\mathrm{H}$ adsorption/desorption peaks. Figure S4 shows a $40 \%$ increase of the AEF with increasing number of de-alloying cycles, with the highest AEF value of $13500 \mathrm{~cm}^{2} / \mathrm{cm}^{2}$ after 50 cycles of de-alloying. This factor is 3 orders of magnitude larger than the one reported for 3D arrays of microstructures (walls, trenches, pillars) using micromachining processes developed in Si microfabrication technology [19-21]. Such large AEF values have also never been reported for DHBT films, indicating the achievement of extremely high porosity from optimized electrodeposition conditions. In order to confirm that the active surface area is as large as suggested by our previous measurements, determination of the AEF was also performed by under potential deposition (UPD) of Cu adatom [22] (Figure S5). In this method, an adatom is adsorbed on the electrode surface to form a monolayer. Then, the adatom is stripped and the charge under the stripping peak is used to determine the active surface area. Figure 
S5a shows the Cu UPD region on Pt DHBT electrode. As the thickness of the Pt DHBT film increases, both the Cu UPD deposition to reach a maximum and the stripping charge increases proportionately (Figure S5b-d). Figure S5e summarizes these results. More importantly, these results showed an excellent correlation with the AEF values determined from $\mathrm{H}$ adsorption/desorption peaks (Figure S5f), thus providing additional confirmation for the extremely large surface area of Pt DHBT electrodes. These high AEF values obtained on Pt DHBT electrodes can be explained by the mechanism of bubble generation and its effect on the growth of the metallic layer. Indeed, the ability of any given metal to catalyze the hydrogen evolution reaction is determined by its exchange current density, jo, and its Tafel slope [5]. As Pt has the highest jo value and low Tafel slope, it generates a high volume of $\mathrm{H}_{2}$ at any specific overpotential, leading to formation of pores with small size, and thus enhanced electrochemically active surface area.

Fig. 1b shows the morphology of Pt DHBT films after de-alloying at different magnifications. The films are globular in shape with cauliflower like morphology, interspersed with mesopores and narrow crevices/nanopores. To understand the crystallographic nature of the Pt/Cu system, we performed GI-XRD at different depths of the film (Figure S6 and Fig. 1c). While going from the surface to deeper regions of the film, the XRD peaks gradually move to lower 2 theta values indicating increase in Pt content. This indicates formation of a substitutional solid solution of $\mathrm{PtCu}$, in line with Vergard's law [23]. This is also logical as during the de-alloying process, majority of the Cu atoms are electrochemically forced to move outward of the film, thus creating a gradient where in Pt rich domains remain at the core and $\mathrm{Cu}$ rich component is more conspicuous in the shell. 
Fig. 1d shows the Pt atomic percentage after de-alloying (obtained from EDX spectra) during increasing deposition time/thickness of the DHBT films. For $600 \mathrm{~s}$ of deposition, the amount of Pt detected before and after de-alloying increases from 60 at. $\%$ to 74 at. \%, indicating successful partial de-alloying of $\mathrm{Cu}$ during the $\mathrm{CV}$ protocol. The final composition is independent on the initial composition of the bath (in the range of the tested $\mathrm{Pt} / \mathrm{Cu}$ bath proportions). This is also reflected in the evolution of $\mathrm{AEF}$ with deposition duration (Fig. 1e), where the same AEF values are obtained independently of the initial Pt:Cu ratios in the electrodeposition bath.

As expected, the AEF increases linearly with the deposition duration and hence the film thickness. Fig. 1f shows the evolution of the AEF normalized by the film thickness. An average value of $93.4 \mathrm{~cm}^{2} / \mathrm{cm}^{2}$ per micron of deposited film is observed, independently of the deposition time. That means that the porous structure of the film does not evolve with its thickness. This observation showcases the versatility of the deposition technique for manufacturing films of varied thickness while retaining extremely large surface area.

\section{2. $\mathrm{RuO}_{2} . \mathrm{xH}_{2} \mathrm{O} / \mathrm{Pt} \mathrm{DHBT}$ electrodes}

We then electrodeposited hydrous $\mathrm{RuO}_{2}$ by cyclic voltammetry on optimized Pt DHBT (Pt:Cu / 67:33, 600 s). In order to simultaneously achieve high surface energy and power density without sacrificing anything in terms of cyclability, it was important to prepare a conformal and continuous deposition of the hydrated $\mathrm{RuO}_{2}$ active material on the surface of the highly structured $\mathrm{Pt}$ electrode. So, the amount of pseudocapacitive material must be large enough to cover the 3D template. However, if the $\mathrm{RuO}_{2}$ deposit is too thick and completely fills the porous structure of the template, the expected benefits of the 3D scaffold will be attenuated and kinetic limitations will 
arise. Fig. 2a shows the $\mathrm{CV}$ profiles of the hydrous $\mathrm{RuO}_{2} / \mathrm{Pt} \mathrm{DHBT}$ electrodes characterized in $0.5 \mathrm{M} \mathrm{H}_{2} \mathrm{SO}_{4}$ solution; the current, $I$, has been normalized by the scan rate, $s$, so that all curves could be shown on the same scale. All CVs exhibit typical pseudocapacitive profiles as expected of hydrous $\mathrm{RuO}_{2}$ with unnoticeable resistive contributions, even at higher scan rates. Interestingly, no peak corresponding to $\mathrm{Cu}$ oxidation is observed in the CVs, due both to the efficiency of the pretreatment leading to the de-alloying of $\mathrm{Cu}$ from the electrode surface, and possibly also to the presence of a conformal hydrous $\mathrm{RuO}_{2}$ deposit that protects the underlying current collector surface. Figure S7 shows the SEM images and EDX mapping of hydrous $\mathrm{RuO}_{2} / \mathrm{Pt}$ DHBT electrodes at different magnifications. The composite electrodes still clearly retain the globular morphology of the initial DHBT framework interspersed with macropores and nonporous crevices evident at low magnifications, suggesting uniform and very thin (in the $\mathrm{nm}$ range) deposition of the hydrous $\mathrm{RuO}_{2}$ layer. XPS studies of the electrodeposited films confirmed the hydrous nature of the deposited $\mathrm{RuO}_{2}$ active materials with characteristic $\mathrm{Ru} 3 \mathrm{~d}, 3 \mathrm{p}_{3 / 2}$ and $\mathrm{O}$ 1s peaks (Figure S8) as reported earlier [24]. The thickness of the hydrous $\mathrm{RuO}_{2}$ layer could be easily controlled by varying the number of CV deposition cycles as exhibited by increasing capacitance values with increasing deposition cycles (Fig. 2b). Extremely high capacitances varying from 7.3 to $20.3 \mathrm{~F} \mathrm{~cm}^{-2}$ were obtained at $1 \mathrm{mV} \mathrm{s}^{-1}$, which are by far the highest values ever reported for a microsupercapacitor electrode $[7,25]$. We then evaluated the ease with which the charge is accessible when the thickness of the hydrous $\mathrm{RuO}_{2}$ layer is increased by calculating the internal and external capacitance as proposed by Trassati et al. [26]. The value of the outer capacitance, $C_{o}$, which is related to the more accessible active surface, reaches $17.1 \mathrm{~F} \mathrm{~cm}^{-2}$ for a total capacitance, $C_{t}$, of $24.2 \mathrm{~F} \mathrm{~cm}^{-}$ 2 for the thickest film (Fig. 2c). This corresponds to an impressive specific energy per 
footprint area of $8.7 \mathrm{~J} \mathrm{~cm}^{-2}$ (i.e. $2.4 \mathrm{mWh} \mathrm{cm}^{-2}$ ). Furthermore, these extraordinarily high capacitance values are combined with decent scan rate capabilities and a high $C_{o}$ contribution of $84 \%$ for 250 cycles of electrodeposition, i.e. most of the stored charge is quickly accessible, fulfilling an important requirement for a supercapacitor. Even the thickest films (750 cycles) have a $C_{o}$ contribution of more than $70 \%$, indicating high power ability of the 3D RuO / Pt DHBT electrodes. Fig. 2d shows the Nyquist plot of the 3D RuO 2 electrode with a perfectly vertical line in the low-frequency region, as expected for pseudocapacitive behaviour, low impedance module value due to the very high capacitance of the electrode, and a very low equivalent series resistance (ESR) of $\sim 0.51 \Omega \mathrm{cm}^{2}$, which corresponds to a specific power of $354 \mathrm{~mW} \mathrm{~cm}-2$. These results demonstrate again that $\mathrm{RuO}_{2} \cdot x \mathrm{H}_{2} \mathrm{O}$ / Pt DHBT microsupercapacitor electrodes, owing to their porous and conductive matrix and large capacitance/ease of charge accessibility, are characterized by both high-energy and high-power density. The composite electrodes also displayed good cycling stability (up to 1600 cycles i.e. 878 hours of ageing) and rate performance (Fig. 2e) with a low ohmic drop of only $12 \mathrm{mV}$ even at the highest current density $\left(10 \mathrm{~mA} \mathrm{~cm}^{-2}\right)$.

\subsection{Self-standing porous oxidized Ru DHBT electrodes}

Inspired by the extremely large capacitance of hydrous $\mathrm{RuO}_{2}$ / Pt DHBT electrodes, we then tried to achieve self-standing porous hydrous $\mathrm{RuO}_{2}$ electrodes. The approach adopted was to first deposit a porous pristine Ru film through the DHBT technique using optimized parameters, followed by the subsequent oxidation of metallic Ru in aqueous medium to make oxidized Ru DHBT electrodes. However, the metallic Ru films showed the same issues with adhesion as those encountered previously with the Pt DHBT deposits. Hence, following the previously established strategy, an 
appropriate amount of $\mathrm{Cu}$ was added to the electrolyte bath as a way to enhance the adherence of the porous films. Fig. 3a shows the de-alloying of $\mathrm{Cu}$ from the composite metallic Ru/Cu DHBT films. The decrease of current between $0.0-0.1 \mathrm{~V}$, indicative of $\mathrm{Cu}$ dissolution, confirmed progressive de-alloying during cycling. This was also confirmed by elemental analysis from EDX measurements, which showed 94 at. \% of the film to be consisting of Ru and only 6 at. \% of $\mathrm{Cu}$ (for Ru/Cu DHBT films before oxidation), thus implying successful de-alloying. Unlike Pt DHBT, the overlap between the hydrogen and oxygen adsorption/desorption peaks on $\mathrm{Ru}$ makes it difficult to determine the electrochemically active surface area (EASA) through estimation of the area under the hydrogen adsorption/desorption peaks [27]. Hence, in case of Ru, the EASA of Ru DHBT was determined by under potential deposition (UPD) of Cu. The procedure followed for the determination of the AEF of de-alloyed Ru DHBT by $\mathrm{Cu}$ UPD is detailed in the Supplementary Material and summarized in Figure S9. The AEF value for porous de-alloyed Ru for 5 minutes of DHBT (74 $\mu \mathrm{m}$ thick) was $\sim 690$ $\mathrm{cm}^{2} / \mathrm{cm}^{2}$. Fig. $3 b$ shows the SEM images of porous de-alloyed Ru films after electrochemical oxidation (see Materials and methods) at different magnifications. The films have typical DHBT type morphology with nanopores interspersed within micropores and a flaky appearance evidenced at the highest magnifications. The chemical nature of the oxidized de-alloyed Ru DHBT films was examined by XPS studies and compared with electrodeposited hydrated $\mathrm{RuO}_{2} \cdot \mathrm{xH}_{2} \mathrm{O}$ and crystalline$\mathrm{RuO}_{2}$ reference obtained from a well-defined $\mathrm{RuO}_{2}(001)$ thin-film that we synthesized by pulsed laser deposition (PLD, Figure S10). Fig. 3c shows the high-resolution Ru 3d spectra of oxidized de-alloyed Ru DHBT films. The Ru $3 d_{5 / 2}$ core level peak appears at relatively higher binding energy values $(281.1 \mathrm{eV})$ and has larger width at half maximum compared to crystalline reference $\mathrm{RuO}_{2}$ (001) (Figure S10a). It also exhibits 
a shoulder at $280.1 \mathrm{eV}$, which can be assigned to a slight remnant of unoxidized metallic Ru. A stark contrast is also noticed in the $\mathrm{O}$ 1s spectra of oxidized de-alloyed $\mathrm{Ru}$ DHBT films (Fig. 3d) as compared to PLD deposited $\mathrm{RuO}_{2}$ (Figure S10c). O 1s peak for oxidized Ru DHBT appears at relatively higher binding energy values (530.9 eV) indicating higher $\mathrm{OH}^{-}$component (from $\mathrm{Ru}-\mathrm{OH}$ ) in the films, whereas the PLD $\mathrm{RuO}_{2}$ films are dominated by $\mathrm{O}^{2-}$ component (from $\mathrm{Ru}-\mathrm{O}-\mathrm{Ru}$ ), thus suggesting hydrous nature of the oxidized de-alloyed Ru DHBT films [24].

The electrochemical performance of oxidized Ru DHBT films was characterized in 0.5 $\mathrm{M} \mathrm{H}_{2} \mathrm{SO}_{4}$ solution in a three-electrode set up. Fig. 3e shows normalized $\mathrm{CV}$ s at different scan rates for a $74 \mu \mathrm{m}$ thick self-standing $\mathrm{Ru}$ DHBT film, showing archetypal pseudocapacitive profiles with negligible change in capacitance as the scan rate is increased from 5 to $50 \mathrm{mV} \mathrm{s}^{-1}$. This was also evident upon deconvolution of the capacitive contribution from 1 to $500 \mathrm{mV} \mathrm{s}^{-1}$ into the outer $C_{0}$ and the total $C_{t}$ capacitance to analyze charge accessibility (Fig. 3f and Figure S11). The outer capacitance $\left(C_{o}=1.8 \mathrm{~F} \mathrm{~cm}^{-2}\right)$ constituted almost $72 \%$ of the total capacitance $\left(C_{t}=\right.$ $2.5 \mathrm{~F} \mathrm{~cm}^{-2}$ ), thus conveying high rate capability and ease of charge access. It is particularly important to note here that compared to Pt DHBT having an AEF > 13000 $\mathrm{cm}^{2} / \mathrm{cm}^{2}$, Ru DHBT film with a relatively "meagre" AEF of $\sim 690 \mathrm{~cm}^{2} / \mathrm{cm}^{2}$ shows an extraordinarily high capacitance of $2.5 \mathrm{~F} \mathrm{~cm}^{-2}$, thanks to only active material matrix without any non-active component and decent electronic conductivity of $\mathrm{RuO}_{2}$.

\subsection{Comparison with existing 3D microsupercapacitor electrodes}

We compared the specific capacitance and AEF values of our porous electrodes with previously reported $3 \mathrm{D}$ electrodes in the literature $[1,6,7,8,28]$ for application in microsupercapacitors. For such microdevices, which are to be integrated into an 
electronic circuitry with limited available space, it is imperative to consider all reported performances normalized to the footprint area on the chip. The $\mathrm{RuO}_{2} / \mathrm{Pt}(\mathrm{Cu}) \mathrm{DHBT}$ electrodes definitely stand miles ahead from most reported studies both in terms of surface area and surface capacitance (Fig. 4), thus making them suitable for loT applications demanding high areal energy density. Accordingly, these microsupercapacitors are excellent contenders for replacing microbatteries, which lack high power performance. Moreover, the minute amount of active material required for the conformal thin film deposition of the porous current collector makes these 3D microsupercapacitor electrodes affordable for microelectronic applications.

Oxidized Ru DHBT electrodes (using the second approach) also show large capacitance even with much lower AEF values. However, they may be restricted for niche applications which have large area/thickness constraints and where cost is not the primary criterion.

Some of the values reported in the literature have been obtained using a relatively high thickness, which may negatively impact their integration into high-resolution interdigitated patterns during the microfabrication process. Since the reported areal capacitance and AEF factor of 3D electrodes may be biased by their thicknesses (see for ex. Fig. 1e), we have also normalized in Figure $\mathrm{S} 12$ the reported specific capacitance and AEF values by the thickness of the 3D electrode for comparison purposes. Independently of the morphology of the underlying porous metallic substrate, the normalized areal capacitance seems to be correlated to the normalized AEF. Regardless of the thickness considered, $\mathrm{RuO}_{2} / \mathrm{Pt}(\mathrm{Cu})$ DHBT shows the highest values of normalized AEF and capacitance, making these electrode materials very promising for the realization of stacked or planar interdigitated microsupercapacitors with the desired dimensions and thickness. 


\section{Conclusion}

In summary, we have successfully demonstrated the design of extremely large surface area porous Pt DHBT current collectors with long-term stability by taking advantage of intrinsic low surface diffusion coefficient of Pt atoms. These porous metallic matrices display exceptionally large AEF values, thus opening up new avenues in applications where the storage of small amount of energy in a restricted surface area is critical. As a proof of concept, electrodeposited hydrous $\mathrm{RuO}_{2}$ on $\mathrm{Pt}$ DHBT resulted in highest ever-reported capacitance, with a record areal energy of $8.7 \mathrm{~J} \mathrm{~cm}^{-2}$. Using a similar strategy, we also demonstrated the fabrication of self-standing porous hydrous $\mathrm{RuO}_{2}$ films with large capacitance, thus emphasizing the versatility of this approach to a wide variety of niche materials requiring large surface area porous films. This is especially interesting since metal-oxide based electrodes are widely utilized as active materials in several areas including batteries, fuel cells, solar cells, pyroelectrics, catalysts, sensors, etc. Moreover, since the entire methodology is based on aqueous electrodeposition, it can be easily scaled up through existing electroplating infrastructure and hence holds extremely high commercial promise.

\section{Acknowledgement}

The authors acknowledge the support from the European Research Council (ERC, Consolidator Grant, ERC-2017-CoG, Project 771793 3D-CAP) and the financial support of the Natural Science and Engineering Council (NSERC). This work was supported by LAAS-CNRS technology platform, a member of Renatech network.

\section{Data availability}


The data that support the plots within this paper can be obtained free of charge from Zenodo via https://zenodo.org.

\section{References}

[1] N.A. Kyeremateng, T. Brousse, D. Pech, Microsupercapacitors as miniaturized energy-storage components for on-chip electronics, Nat. Nanotechnol. 12 (2017) 7-15, https://doi.org/10.1038/nnano.2016.196.

[2] L. Liu, H. Zhao, Y. Lei, Advances on three-dimensional electrodes for micro-supercapacitors: A mini-review, InfoMat. 1 (2019) 74-84, https://doi.org/10.1002/inf2.12007.

[3] V. Augustyn, P. Simon, B. Dunn, Pseudocapacitive oxide materials for high-rate electrochemical energy storage, Energy Environ. Sci. 7 (2014) 1597-1614, https://doi.org/10.1039/c3ee44164d.

[4] K. Brousse, S. Pinaud, S. Nguyen, P.-F. Fazzini, R. Makarem, C. Josse, Y. Thimont, B. Chaudret, P.-L. Taberna, M. Respaud, P. Simon, Facile and scalable preparation of ruthenium oxide-based flexible micro-supercapacitors, Adv. Energy Mater. 10 (2020) 1903136, https://doi.org/10.1002/aenm.201903136.

[5] B.J. Plowman, L.A. Jones, S.K. Bhargava, Building with bubbles: the formation of high surface area honeycomb-like films via hydrogen bubble templated electrodeposition, Chem. Commun. 51 (2015) 4331-4346, https://doi.org/10.1039/C4CC06638C.

[6] A. Ferris, S. Garbarino, D. Guay, D. Pech, 3D $\mathrm{RuO}_{2}$ Microsupercapacitors with remarkable areal energy, Adv. Mater. 27 (2015) 6625-6629, https://doi.org/10.1002/adma.201503054.

[7] S.G. Patnaik, J. Shamsudeen Seenath, D. Bourrier, S. Prabhudev, D. Guay, D. Pech, Porous $\mathrm{RuO}_{x} \mathrm{~N}_{y} \mathrm{~S}_{z}$ electrodes for microsupercapacitors and microbatteries with enhanced areal performance, ACS Energy Lett. 6 (2021) 131-139,. https://doi.org/10.1021/acsenergylett.0c02017.

[8] A. Ferris, D. Bourrier, S. Garbarino, D. Guay, D. Pech, 3D interdigitated microsupercapacitors with record areal cell capacitance, Small 15 (2019) 1901224, https://doi.org/10.1002/smll.201901224. [9] S.G. Patnaik, A. Jadon, C.C.H. Tran, A. Estève, D. Guay, D. Pech, High areal capacity porous SnAu alloys with long cycle life for Li-ion microbatteries, Sci. Rep. 10 (2020) 1-8, https://doi.org/10.1038/s41598-020-67309-7. 
[10] S.G. Patnaik, D. Pech, Low temperature deposition of highly cyclable porous Prussian blue cathode for lithium-ion microbattery, Small 17 (2021) 20101615, https://doi.org/10.1002/smll.202101615.

[11] J. Snyder, P. Asanithi, A.B. Dalton, J. Erlebacher, Stabilized nanoporous metals by dealloying ternary alloy precursors, Adv. Mater. 20 (2008) 4883-4886, https://doi.org/10.1002/adma.200702760. [12] S. Cherevko, N. Kulyk, C.H. Chung, Nanoporous Pt@Au $\mathrm{Au}_{100-x}$ by hydrogen evolution assisted electrodeposition of $\mathrm{Au}_{x} \mathrm{Cu}_{100-x}$ and galvanic replacement of $\mathrm{Cu}$ with Pt: Electrocatalytic properties, Langmuir 28 (2012) 3306-3315, https://doi.org/10.1021/la203625e.

[13] S. Trasatti, O.A. Patrii, Real surface area measurements in electrochemistry. J. Electroanal. Chem. 327 (1992) 353-376, https://doi.org/10.1016/0022-0728(92)80162-W.

[14] C.L. Green, A. Kucernak, Determination of the platinum and ruthenium surface areas in platinumruthenium alloy electrocatalysts by underpotential deposition of copper. I. Unsupported Catalysts. J. Phys. Chem. B 106 (2002) 1036-1047, https://doi.org/10.1021/ip0131931.

[15] D.V. Pugh, A. Dursun, S.G. Corcoran, Electrochemical and morphological characterization of PtCu dealloying, J. Electrochem. Soc. 152 (2005) B455, https://doi.org/10.1149/1.2048140.

[16] X. Lang, A. Hirata, T. Fujita, M. Chen, Nanoporous metal/oxide hybrid electrodes for electrochemical supercapacitors, Nat. Nanotechnol. 6 (2011) 232-236, https://doi.org/10.1038/nnano.2011.13.

[17] Z. Luo, J. Xu, B. Yuan, R. Hu, L. Yang, Y. Gao, M. Zhu, 3D hierarchical porous Cu-based composite current collector with enhanced ligaments for notably improved cycle stability of Sn anode in Li-ion batteries, ACS Appl. Mater. Interfaces. 10 (2018) 22050-22058, https://doi.org/10.1021/acsami.8b04049.

[18] Y. Yu, L. Gu, X. Lang, C. Zhu, T. Fujita, M. Chen, J. Maier, Li Storage in 3D Nanoporous AuSupported Nanocrystalline Tin, Adv. Mater. 23 (2011) 2443-2447, https://doi.org/10.1002/adma.201004331.

[19] E. Eustache, P. Tilmant, L. Morgenroth, P. Roussel, G. Patriarche, D. Troadec, N. Rolland, T. Brousse, C. Lethien, Silicon-microtube scaffold decorated with anatase $\mathrm{TiO} 2$ as a negative electrode for a 3D lithium-ion microbattery, Adv. Energy Mater. 4 (2014) 1301612, https://doi.org/10.1002/aenm.201301612. 
[20] E. Eustache, C. Douard, R. Retoux, C. Lethien, T. Brousse, MnO2 thin film on 3D scaffold: microsupercapacitor electrodes competing with "bulk" carbon electrodes, Adv. Energy Mater. 5 (2015) 1500680, https://doi.org/10.1002/aenm.201500680.

[21] M. Létiche, E. Eustache, J. Freixas, A. Demortière, V. de Andrade, L. Morgenroth, P. Tilmant, F. Vaurette, D. Troadec, P. Roussel, T. Brousse, C. Lethien, Atomic layer deposition of functional layers for on chip 3D Li-ion all solid state microbattery, Adv. Energy Mater. 7 (2017) 1601402,

\section{https://doi.org/10.1002/aenm.201601402.}

[22] D. Chen, Q. Tao, L.W. Liao, S.X. Liu, Y.X. Chen, S. Ye, Determining the active surface area for various platinum electrodes, Electrocatalysis 2 (2011) 207-219, https://doi.org/10.1007/s12678-011$\underline{0054-1 .}$

[23] X. Zhao, B. Luo, R. Long, C. Wang, Y. Xiong, Composition-dependent activity of Cu-Pt alloy nanocubes for electrocatalytic $\mathrm{CO}_{2}$ reduction, J. Mater. Chem. A. 3 (2015) 4134-4138, https://doi.org/10.1039/c4ta06608a.

[24] D. Rochefort, P. Dabo, D. Guay, P.M.A. Sherwood, XPS investigations of thermally prepared $\mathrm{RuO}_{2}$ electrodes in reductive conditions, Electrochim. Acta. 48 (2003) 4245-4252, https://doi.org/10.1016/S0013-4686(03)00611-X.

[25] B. Asbani, G. Buvat, J. Freixas, M. Huvé, D. Troadec, P. Roussel, T. Brousse, C. Lethien, Ultrahigh areal capacitance and high rate capability $\mathrm{RuO}_{2}$ thin film electrodes for 3D micro-supercapacitors, Energy Stor. Mater. 42 (2021) 259-267, https://doi.org/10.1016/j.ensm.2021.07.038.

[26] S. Ardizzone, G. Fregonara, S. Trasatti, "Inner" and "outer" active surface of $\mathrm{RuO}_{2}$ electrodes, Electrochim. Acta 35 (1990) 263-267, https://doi.org/10.1016/0013-4686(90)85068-X.

[27] T. Nagel, N. Bogolowski, H. Baltruschat, Towards a determination of the active surface area of polycrystalline and nanoparticle electrodes by $\mathrm{Cu}$ upd and $\mathrm{CO}$ oxidation, J. Appl. Electrochem. 36 (2006) 1297-1306, https://doi.org/10.1007/s10800-006-9187-y.

[28] B. Bounor, B. Asbani, C. Douard, F. Favier, T. Brousse, C. Lethien, On chip $\mathrm{MnO}_{2}$-based 3D micro-supercapacitors with ultra-high areal energy density, Energy Stor. Mater. 38 (2021) 520-527, https://doi.org/10.1016/j.ensm.2021.03.034. 


\section{FIGURE 1}

a

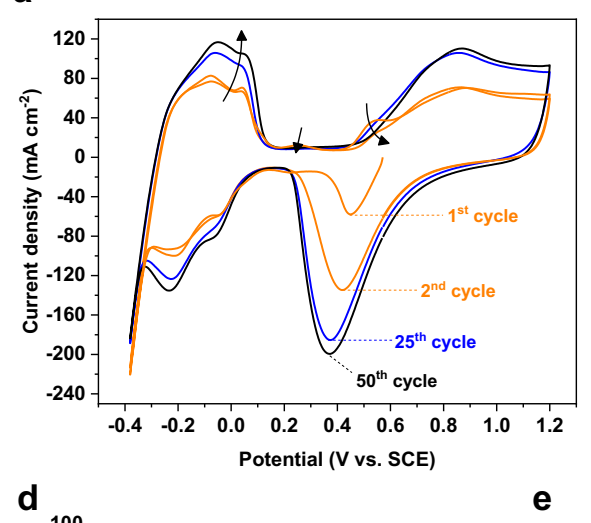

d

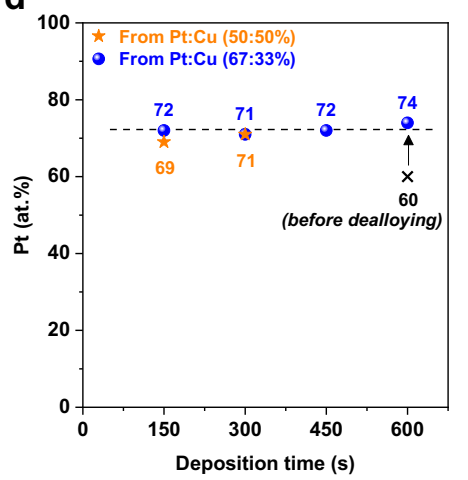

b

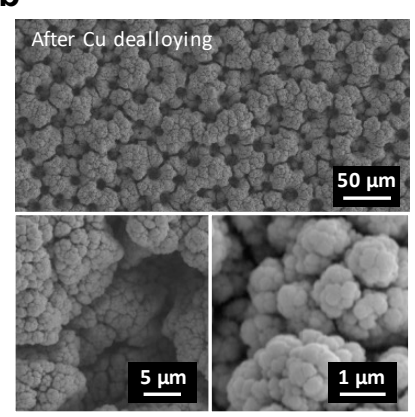

e

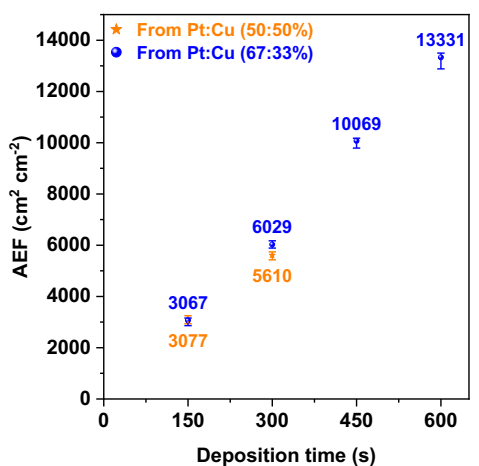

C

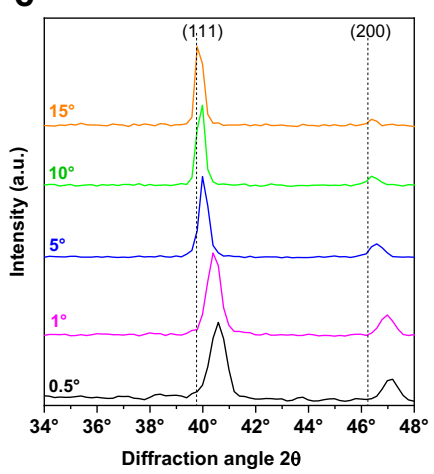

f

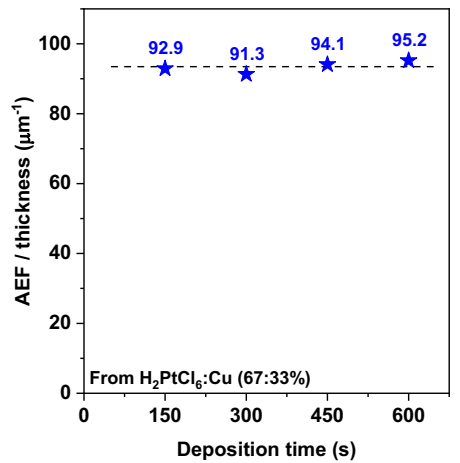

Fig. 1. Synthesis and characterizations of Pt DHBT current collectors. a. CV evolution of Cu de-alloying at $10 \mathrm{mV} \mathrm{s}^{-1}$ in $0.5 \mathrm{M} \mathrm{H}_{2} \mathrm{SO}_{4}$. b. SEM images of de-alloyed Pt DHBT. c. De-alloyed Pt DHBT (111) and (200) diffraction peaks at different grazing incidences angles. d. Pt atomic percentage of DHBT scaffold after $\mathrm{Cu}$ de-alloying for different deposition time and bath composition of $\mathrm{H}_{2} \mathrm{PtCl}_{6}$ and $\mathrm{CuSO}_{4} \cdot 5 \mathrm{H}_{2} \mathrm{O}$. e. Evolution of $\mathrm{AEF}$ with the DHBT deposition time. f. Thickness-normalized AEF with deposition time. 


\section{FIGURE 2}

a
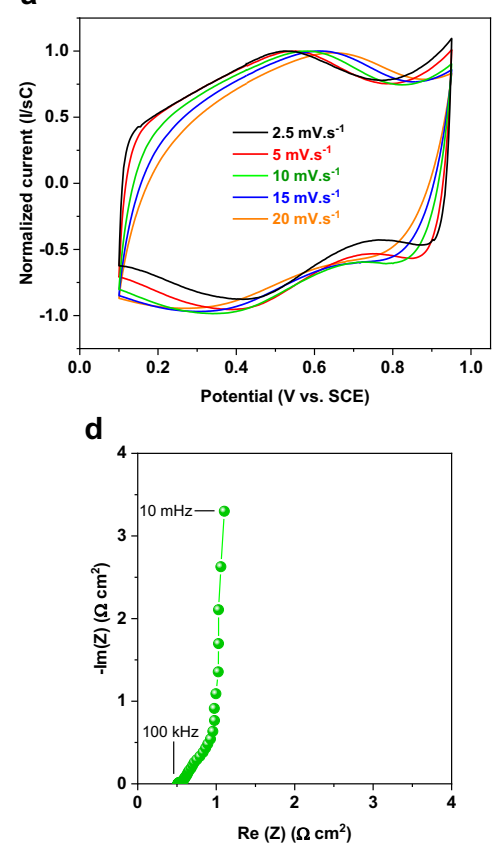

b

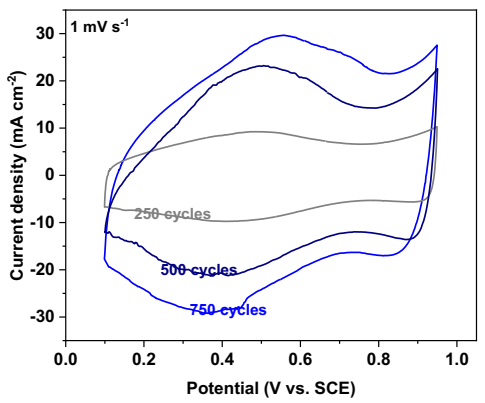

C

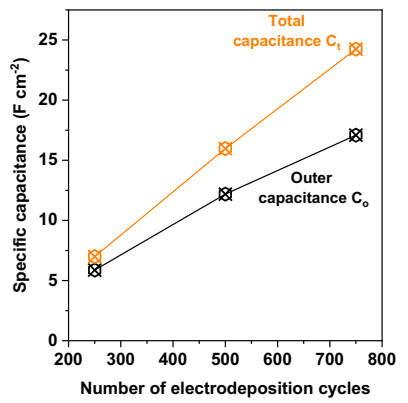

e

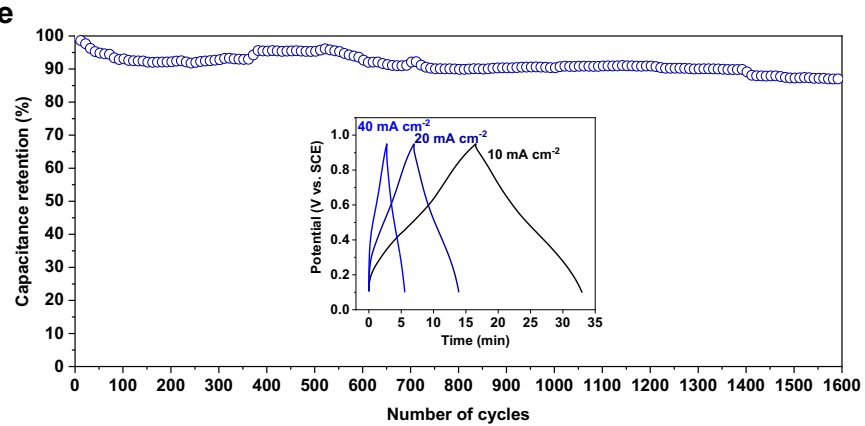

Fig. 2. Electrochemical characterizations of $\mathrm{RuO}_{2} \cdot \mathrm{xH}_{2} \mathrm{O} / \mathrm{Pt}$ DHBT electrodes. a. Normalized CVs at different scan rates in $0.5 \mathrm{M} \mathrm{H}_{2} \mathrm{SO}_{4}$. b. $\mathrm{CVs}$ evolution at $1 \mathrm{mV} \mathrm{s}^{-1}$ with increasing number of electrodeposition cycles of $\mathrm{RuO}_{2} \cdot \mathrm{xH}_{2} \mathrm{O}$ onto Pt DHBT. c. Evolution of the total and outer capacitance with the number of electrodeposition cycles. d. Nyquist plot of $\mathrm{RuO}_{2} \cdot \mathrm{xH}_{2} \mathrm{O}$ / Pt DHBT electrode. e. Stability of the electrode during long-term galvanostatic charge-discharge studies and corresponding potential time profiles at different applied current densities. 


\section{FIGURE 3}

a

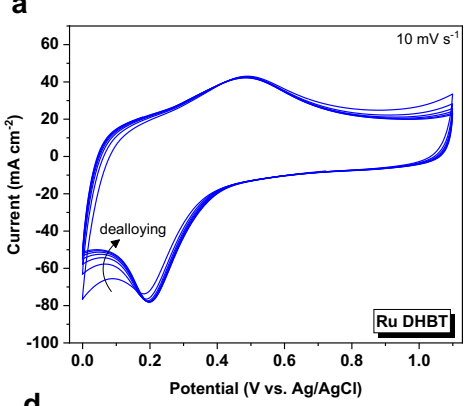

d

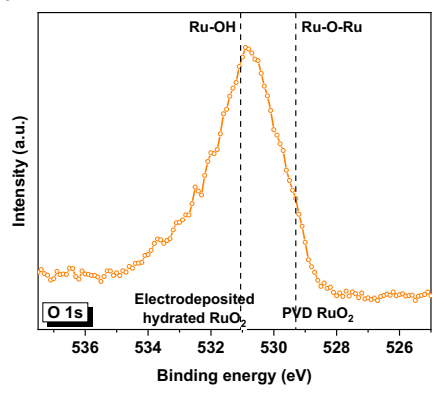

b

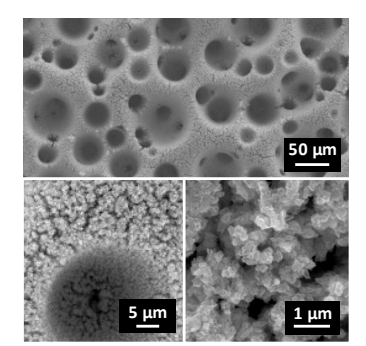

e

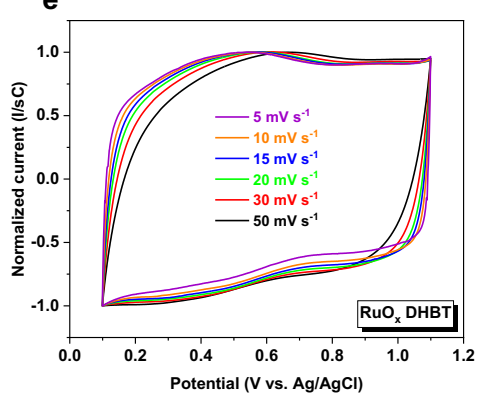

C

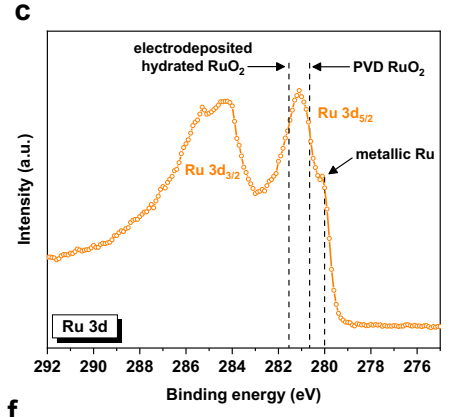

f

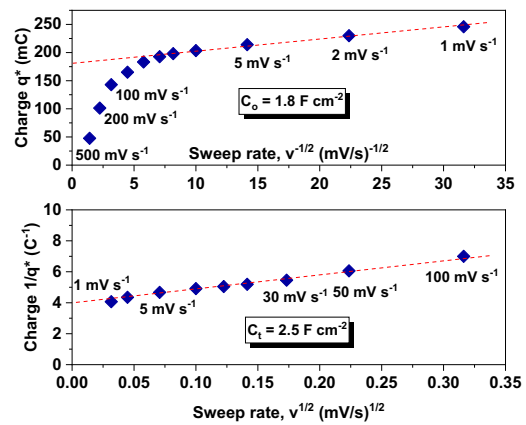

Fig. 3. Synthesis and characterizations of oxidized de-alloyed Ru DHBT electrodes. a. Cu de-alloying CVs of Ru DHBT in $0.5 \mathrm{M} \mathrm{H}_{2} \mathrm{SO}_{4}$. b. SEM images of oxidized de-alloyed Ru DHBT. c. Ru 3d core-level XPS spectrum of oxidized de-alloyed Ru DHBT. d. O 1s core-level XPS spectrum. e. Normalized CVs at different scan rates in $0.5 \mathrm{M} \mathrm{H}_{2} \mathrm{SO}_{4}$. f. Determination of the outer $\left(C_{o}\right)$ and total capacitance $\left(C_{t}\right)$ of the self-standing porous electrode. 


\section{FIGURE 4}

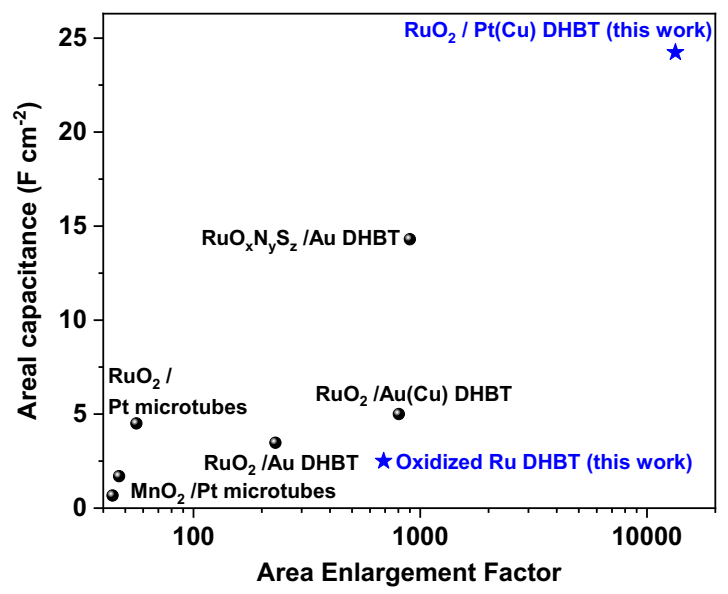

Fig. 4. Comparison with state-of-the-art 3D electrode materials for microsupercapacitors: areal capacitance vs. reported AEF values for different 3D architectures. 